Bull. Korean Math. Soc. 48 (2011), No. 1, pp. 9-15

DOI 10.4134/BKMS.2011.48.1.009

\title{
ON THE $2 k$-TH POWER MEAN VALUE OF THE GENERALIZED QUADRATIC GAUSS SUMS
}

\author{
YANFEng He AND Wenpeng Zhang
}

\begin{abstract}
The main purpose of this paper is using the elementary and analytic methods to study the properties of the $2 k$-th power mean value of the generalized quadratic Gauss sums, and give two exact mean value formulae for $k=3$ and 4 .
\end{abstract}

\section{Introduction}

Let $q \geq 2$ be an integer, $\chi$ denotes a Dirichlet character modulo $q$. For any integer $n$, we define the generalized quadratic Gauss sums $G(n, \chi ; q)$ as follows:

$$
G(n, \chi ; q)=\sum_{a=1}^{q} \chi(a) e\left(\frac{n a^{2}}{q}\right)
$$

where $e(y)=e^{2 \pi i y}$. This sum is important, because it is a generalization of the classical quadratic Gauss sums $G(n, q)$, which is defined by

$$
G(n ; q)=\sum_{a=1}^{q} e\left(\frac{n a^{2}}{q}\right) .
$$

About the properties of $G(n, \chi ; q)$, some authors had studied it, and obtained many interesting results. For example, for any integer $n$ with $(n, q)=1$, from the general result of Cochrane and Zheng [2] we can deduce that

$$
|G(n, \chi ; q)| \leq 2^{\omega(q)} q^{\frac{1}{2}},
$$

where $\omega(q)$ denotes the number of all distinct prime divisors of $q$. The case where $q$ is a prime is due to Weil [4]. Zhang [5] proved that for any odd prime $p$ and integer $n$ with $(n, p)=1$, we have

$$
\sum_{\chi \bmod p}|G(n, \chi ; p)|^{4}= \begin{cases}(p-1)\left[3 p^{2}-6 p-1+4\left(\frac{n}{p}\right) \sqrt{p}\right], & \text { if } p \equiv 1 \bmod 4 \\ (p-1)\left(3 p^{2}-6 p-1\right), & \text { if } p \equiv 3 \bmod 4\end{cases}
$$

Received April 6, 2009.

2010 Mathematics Subject Classification. Primary 11M20.

Key words and phrases. generalized quadratic Gauss sums, $2 k$-th power mean value, calculating formula, identity.

This work is supported by the N. S. F. (10671155) of P. R. China. 
and

$$
\sum_{\chi \bmod p}|G(n, \chi ; p)|^{6}=(p-1)\left(10 p^{3}-25 p^{2}-4 p-1\right), \text { if } p \equiv 3 \bmod 4,
$$

where $\left(\frac{n}{p}\right)$ is the Legendre symbol.

Besides, W. Zhang and H. Liu [6] also proved the following conclusion:

Let $q \geq 3$ be a square-full number. Then for any integers $n, k$ with $(n k, q)=$ 1 and $k \geq 1$, we have the identity

$$
\sum_{\chi \bmod q}|G(n, \chi ; q)|^{4}=q \cdot \phi^{2}(q) \prod_{p \mid q}(k, p-1)^{2} \prod_{\substack{p \mid q \\(k, p-1)=1}} \frac{\phi(p-1)}{p-1},
$$

where $\prod_{p \mid q}$ denotes the product over all prime divisors of $q, \phi(q)$ is the Euler function.

In this paper, we use the elementary and analytic methods to study the calculating problem of the $2 k$-th power mean value of the generalized quadratic Gauss sums, and give two exact calculating formulae for $k=3$ and 4 . That is, we shall prove the following:

Theorem 1. Let odd number $q>1$ be a square-full number (i.e., for any prime $p, p \mid q$ if and only if $\left.p^{2} \mid q\right)$. Then for any integer $n$ with $(n, q)=1$, we have the identity

$$
\sum_{\chi \bmod q}|G(n, \chi ; q)|^{6}=16^{\omega(q)} \cdot q^{2} \cdot \phi^{2}(q),
$$

where $\omega(q)$ denotes the number of all distinct prime divisors of $q$.

Theorem 2. Let odd number $q>1$ be a square-full number. Then for any integer $n$ with $(n, q)=1$, we have

$$
\sum_{\chi \bmod q}|G(n, \chi ; q)|^{8}=64^{\omega(q)} \cdot q^{3} \cdot \phi^{2}(q) .
$$

From our theorems we know that the estimates in reference [2] is the best one. In fact from Theorem 2 we know that there exists at least one Dirichlet character modulo $q$ such that the inequality:

$$
|G(n, \chi ; q)| \geq 2^{\frac{3}{4} \omega(q)} q^{\frac{3}{8}} \phi^{\frac{1}{8}}(q) .
$$

For general integer $k \geq 5$, we believe that the following conclusion is correct:

Conjecture. Let odd number $q>1$ be a square-full number, $k \geq 2$ be an integer. Then for any integer $n$ with $(n, q)=1$, we have the identity

$$
\sum_{\chi \bmod q}|G(n, \chi ; q)|^{2 k}=4^{(k-1) \omega(q)} \cdot q^{k-1} \cdot \phi^{2}(q) .
$$

The proposed method is supposed to be capable of proving this formula. However, the calculation will be so complex when $k \geq 5$ that such a general 
conclusion cannot be obtained. For general positive integer $q>3$, it is an open problem whether there is a formula to calculate the $2 k$-th power mean value of the generalized quadratic Gauss sums.

\section{Several lemmas}

To complete the proof of our theorems, we need the following several lemmas.

Lemma 1. For any integer $q \geq 1$, we have the identity

$$
G(1 ; q)=\frac{1}{2} \sqrt{q}(1+i)\left(1+e^{\frac{-\pi i q}{2}}\right)=\left\{\begin{array}{lll}
\sqrt{q}, & \text { if } q \equiv 1 & (\bmod 4) \\
0, & \text { if } q \equiv 2 & (\bmod 4) \\
i \sqrt{q}, & \text { if } q \equiv 3 & (\bmod 4) ; \\
(1+i) \sqrt{q}, & \text { if } q \equiv 0 & (\bmod 4)
\end{array}\right.
$$

Proof. This is a remarkable formula of Gauss. See Theorem 9.16 of [1].

Lemma 2. Let $p$ be an odd prime and $\alpha \geq 2$ be an integer. Then for any integer $n$ with $(p, n)=1$, we have the identity

$$
\sum_{b=1}^{p^{\alpha}} e\left(\frac{n b^{2}}{p^{\alpha}}\right)=0
$$

Proof. First we know that for any positive integers $q \geq 2$ and integer $n$ with $(n, q)=1$, we have the identity

$$
\sum_{u=0}^{q-1} e\left(\frac{u n}{q}\right)=0
$$

From this identity and the properties of reduce residue system we have

$$
\begin{aligned}
\sum_{b=1}^{p^{\alpha}} e\left(\frac{n b^{2}}{p^{\alpha}}\right) & =\sum_{u=0}^{p-1} \sum_{v=1}^{p^{\alpha-1}} e\left(\frac{n\left(u p^{\alpha-1}+v\right)^{2}}{p^{\alpha}}\right)=\sum_{u=0}^{p-1} \sum_{v=1}^{p^{\alpha-1}} e\left(\frac{2 n u v p^{\alpha-1}+v^{2}}{p^{\alpha}}\right) \\
& =\sum_{v=1}^{p^{\alpha-1}} e\left(\frac{v^{2}}{p^{\alpha}}\right) \sum_{u=0}^{p-1} e\left(\frac{2 n u v}{p}\right)=0 .
\end{aligned}
$$

This proves Lemma 2 .

Lemma 3. Let $m, n \geq 2$ and $u$ be three integers with $(m, n)=1$ and $(u, m n)=$ 1. Then for any character $\chi=\chi_{1} \chi_{2}$ with $\chi_{1} \bmod m$ and $\chi_{2} \bmod n$, we have the identity

$$
G(u, \chi ; m n)=\chi_{1}(n) \chi_{2}(m) G\left(u n, \chi_{1} ; m\right) G\left(u m, \chi_{2} ; n\right) .
$$

Proof. See Lemma 6 of [6].

Lemma 4. Let $p$ be an odd prime, $\alpha \geq 2$ and $n$ be two integers with $(n, p)=1$.

Then we have

$$
\sum_{\chi \bmod p^{\alpha}}\left|G\left(n, \chi ; p^{\alpha}\right)\right|^{6}=16 \phi^{2}\left(p^{\alpha}\right) p^{2 \alpha} .
$$


Proof. From the definition of $G\left(n, \chi ; p^{\alpha}\right)$ we have

$$
\begin{aligned}
\left|G\left(n, \chi ; p^{\alpha}\right)\right|^{2} & =\sum_{a=1}^{p^{\alpha}} \sum_{b=1}^{p^{\alpha}} \chi(a) \bar{\chi}(b) e\left(\frac{n\left(a^{2}-b^{2}\right)}{p^{\alpha}}\right) \\
& =\sum_{a=1}^{p^{\alpha}} \chi(a) \sum_{b=1}^{p^{\alpha}} e\left(\frac{n b^{2}\left(a^{2}-1\right)}{p^{\alpha}}\right) .
\end{aligned}
$$

Then by this formula and the orthogonality relation for character sums modulo $p^{\alpha}$ we may get

$$
\begin{aligned}
& \sum_{\chi \bmod p^{\alpha}}\left|G\left(n, \chi ; p^{\alpha}\right)\right|^{6} \\
= & \phi\left(p^{\alpha}\right) \sum_{\substack{a=1 \\
a b c \equiv 1}}^{p^{\alpha}} \sum_{\substack{b^{\alpha} \\
p^{\alpha}}}^{\prime} \sum_{c=1}^{p^{\alpha}}\left(\sum_{u=1}^{\prime} e\left(\frac{n u^{2}\left(a^{2}-1\right)}{p^{\alpha}}\right)\right) \\
\times & \left(\sum_{v=1}^{p^{\alpha}} e\left(\frac{n v^{2}\left(b^{2}-1\right)}{p^{\alpha}}\right)\right)\left(\sum_{w=1}^{p^{\alpha}} e\left(\frac{n w^{2}\left(c^{2}-1\right)}{p^{\alpha}}\right)\right) .
\end{aligned}
$$

Let $\left(a^{2}-1, p^{\alpha}\right)=p^{m}$. If $m \leq \alpha-2$, note that $\left(n\left(a^{2}-1\right) / p^{m}, p\right)=1$, then from Lemma 2 we have

$$
\sum_{u=1}^{p^{\alpha}} e\left(\frac{n u^{2}\left(a^{2}-1\right)}{p^{\alpha}}\right)=p^{m} \sum_{u=1}^{p^{\alpha-m}} e\left(\frac{n u^{2}\left(a^{2}-1\right) / p^{m}}{p^{\alpha-m}}\right)=0 .
$$

If $m=\alpha$, then

$$
\sum_{u=1}^{p^{\alpha}} e\left(\frac{n u^{2}\left(a^{2}-1\right)}{p^{\alpha}}\right)=\phi\left(p^{\alpha}\right) .
$$

If $m=\alpha-1$, then $a=r p^{\alpha-1} \pm 1,1 \leq r \leq p-1$. Note that for any prime $p$ with $p \nmid n$, by Theorem 7.5.4 of [3] we have

$$
G(n ; p)=\left(\frac{n}{p}\right) G(1 ; p) .
$$

Then from (2) and Lemma 1 we may get

$$
\begin{aligned}
\sum_{u=1}^{p^{\alpha}} e\left(\frac{n u^{2}\left(a^{2}-1\right)}{p^{\alpha}}\right) & =p^{\alpha-1} \sum_{u=1}^{p} e\left(\frac{n u^{2}\left(a^{2}-1\right) / p^{\alpha-1}}{p}\right) \\
& =p^{\alpha-1}\left[\left(\frac{ \pm 2 r n}{p}\right) G(1 ; p)-1\right] .
\end{aligned}
$$

Note that the number of the solutions of the congruent equation $1 \leq a, b, c \leq$ $p^{\alpha}-1$ with $p^{\alpha}\left|a^{2}-1, p^{\alpha}\right| b^{2}-1, p^{\alpha} \mid c^{2}-1$ and $a b c \equiv 1 \bmod p^{\alpha}$ are 4 , the 
number of the solutions of the congruent equation $1 \leq a, b, c \leq p^{\alpha}-1$ with $p^{\alpha}\left|a^{2}-1, p^{\alpha}\right| b^{2}-1, p^{\alpha-1} \| c^{2}-1$ and $a b c \equiv 1 \bmod p^{\alpha}$ are 0 , and

$$
\begin{aligned}
& \sum_{\substack{a=1 \\
p^{\alpha} \mid a^{2}-1}}^{p^{\alpha}} \sum_{\substack{b=1 \\
p^{\alpha-1} \| b^{2}-1}}^{p^{\alpha}} \sum_{\substack{c=1 \\
p^{\alpha-1} \| c^{2}-1}}^{p^{\alpha}}\left(\sum_{u=1}^{p^{\alpha}} e\left(\frac{n u^{2}\left(a^{2}-1\right)}{p^{\alpha}}\right)\right) \\
& a b c \equiv 1 \bmod p^{\alpha} \\
& \times\left(\sum_{v=1}^{p^{\alpha}} e\left(\frac{n v^{2}\left(b^{2}-1\right)}{p^{\alpha}}\right)\right)\left(\sum_{w=1}^{p^{\alpha}} e\left(\frac{n w^{2}\left(c^{2}-1\right)}{p^{\alpha}}\right)\right) \\
& =\phi\left(p^{\alpha}\right) \sum_{\substack{a=1 \\
p^{\alpha} \mid a^{2}-1}}^{p^{\alpha}} \sum_{\substack{b=1 \\
p^{\alpha-1} \| b^{2}-1 \\
a b c \equiv 1 \bmod p^{\alpha}}}^{p^{\alpha}} \sum_{\substack{c=1 \\
p^{\alpha-1} \| c^{2}-1}}^{p^{\alpha}}\left(\sum_{v=1}^{p^{\alpha}} e\left(\frac{n v^{2}\left(b^{2}-1\right)}{p^{\alpha}}\right)\right)\left(\sum_{w=1}^{p^{\alpha}} e\left(\frac{n w^{2}\left(c^{2}-1\right)}{p^{\alpha}}\right)\right) \\
& =4 \phi\left(p^{\alpha}\right) p^{2(\alpha-1)} \sum_{r=1}^{p-1}\left[\left(\frac{-1}{p}\right) G^{2}(1 ; p)+1\right] \\
& -4 \phi\left(p^{\alpha}\right) p^{2(\alpha-1)} \sum_{r=1}^{p-1}\left[\left(\frac{-2 r n}{p}\right)+\left(\frac{2 r n}{p}\right)\right] G(1 ; p) \\
& =4 \phi\left(p^{\alpha}\right) p^{2(\alpha-1)}\left(p^{2}-1\right) \text {. }
\end{aligned}
$$

So combining the above several cases and (1) we have

$$
\begin{aligned}
& \sum_{\bmod p^{\alpha}}\left|G\left(n, \chi ; p^{\alpha}\right)\right|^{6} \\
= & 4 \phi^{4}\left(p^{\alpha}\right)+12 \phi^{2}\left(p^{\alpha}\right) p^{2(\alpha-1)}\left(p^{2}-1\right) \\
& +\phi\left(p^{\alpha}\right) p^{3(\alpha-1)}\left[\left(\frac{ \pm 2 r n}{p}\right) G(1 ; p)-1\right] \\
& \times\left[\left(\frac{ \pm 2 s n}{p}\right) G(1 ; p)-1\right]\left[\left(\frac{ \pm 2 t n}{p}\right) G(1 ; p)-1\right] \\
= & 4 \phi^{4}\left(p^{\alpha}\right)+12 \phi^{2}\left(p^{\alpha}\right) p^{2(\alpha-1)}\left(p^{2}-1\right) \\
& +\phi\left(p^{\alpha}\right) p^{3(\alpha-1)} \sum_{\substack{r=1 \\
(r-1}}^{p-1} \sum_{s=1}^{p-1} \sum_{t=1}^{p-1}\left[\left(\frac{2 r n}{p}\right) G(1 ; p)-1\right] \\
& \times\left[\left(\frac{2 s n}{p}\right) G(1 ; p)-1\right]\left[\left(\frac{2 t n}{p}\right) G(1 ; p)-1\right] \\
& +3 \phi\left(p^{\alpha}\right) p^{3(\alpha-1)} \sum_{r=1}^{p-1} \sum_{s=1}^{p-1} \sum_{\substack{t=1 \\
s+t \equiv r}}^{p-1}\left[\left(\frac{2 r n}{p}\right) G(1 ; p)-1\right]
\end{aligned}
$$




$$
\begin{aligned}
& \times\left[\left(\frac{-2 s n}{p}\right) G(1 ; p)-1\right]\left[\left(\frac{-2 t n}{p}\right) G(1 ; p)-1\right] \\
&= 4 \phi^{4}\left(p^{\alpha}\right)+12 \phi^{2}\left(p^{\alpha}\right) p^{2(\alpha-1)}\left(p^{2}-1\right) \\
&+4 \phi\left(p^{\alpha}\right) p^{3(\alpha-1)} \sum_{\substack{r=1 \\
r+s+t=0}}^{p-1} \sum_{\substack{s=1 \\
r+s=1}}^{p-1}\left[\left(\frac{2 r n}{p}\right) G(1 ; p)-1\right] \\
&(3) \quad \times\left[\left(\frac{2 s n}{p}\right) G(1 ; p)-1\right]\left[\left(\frac{2 t n}{p}\right) G(1 ; p)-1\right] .
\end{aligned}
$$

From the properties of the Legendre symbol (see reference [1]) we know that

(4)

$$
\begin{aligned}
& \sum_{\substack{r=1 \\
r+s+t=0}}^{p-1} \sum_{\substack{s=1 \\
r=1}}^{p-1}\left(\frac{r}{p}\right)\left(\frac{s}{p}\right)\left(\frac{t}{p}\right)=\sum_{r=1}^{p-1} \sum_{s=1}^{p-1}\left(\frac{\mp r s(r+s)}{p}\right) \\
& =\sum_{r=1}^{p-1} \sum_{s=1}^{p-1}\left(\frac{\mp r s^{2}(r s+s)}{p}\right)=\sum_{r=1}^{p-1}\left(\frac{\mp r(r+1)}{p}\right) \sum_{s=1}^{p-1}\left(\frac{s}{p}\right)=0, \\
& \sum_{\substack{r=1 \\
r+s \pm t \equiv 0}}^{p-1} \sum_{\substack{s=1 \\
\bmod p}}^{p-1} \sum_{\substack{t=1 \\
p}}^{p-1}\left(\frac{r}{p}\right)\left(\frac{s}{p}\right)=\sum_{r=1}^{p-1} \sum_{s=1}^{p-1}\left(\frac{r s}{p}\right)-\sum_{\substack{r=1 \\
r+s \equiv 0 \bmod p}}^{p-1} \sum_{s=1}^{p-1}\left(\frac{r s}{p}\right) \\
& \text { (5) } \quad=-\sum_{r=1}^{p-1}\left(\frac{-r^{2}}{p}\right)=-\left(\frac{-1}{p}\right)(p-1) \text {, }
\end{aligned}
$$

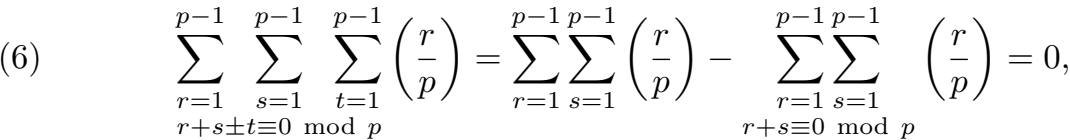

$$
\begin{aligned}
& \sum_{\substack{r=1 \\
r+s \pm t \equiv 0}}^{p-1} \sum_{\substack{s=1 \\
\bmod p}}^{p-1} \sum_{\substack{t=1 \\
\operatorname{mos}}}^{p-1} \sum_{r=1}^{p-1} \sum_{s=1}^{p-1} 1-\sum_{\substack{r=1 \\
r+s \equiv 0 \bmod p}}^{p-1} \sum_{s=1}^{p-1}=(p-1)(p-2) .
\end{aligned}
$$

Note that $\left(\frac{-1}{p}\right) G^{2}(1 ; p)=p$, from (3), (4), (5), (6) and (7) we may get

$$
\begin{aligned}
& \sum_{\chi \bmod p^{\alpha}}\left|G\left(n, \chi ; p^{\alpha}\right)\right|^{6}=4 \phi^{4}\left(p^{\alpha}\right)+12 \phi^{2}\left(p^{\alpha}\right) p^{2(\alpha-1)}\left(p^{2}-1\right) \\
&+12 \phi^{2}\left(p^{\alpha}\right) p^{2 \alpha-1}-4 \phi^{2}\left(p^{\alpha}\right) p^{2(\alpha-1)}(p-2) \\
&=16 \phi^{2}\left(p^{\alpha}\right) p^{2 \alpha} .
\end{aligned}
$$

This proves Lemma 4. 


\section{Proof of theorems}

In this section, we shall complete the proof of our theorems. We only prove Theorem 1. Similarly, we can deduce Theorem 2. In fact if $q$ is an odd squarefull number, let $q=p_{1}^{\alpha_{1}} p_{2}^{\alpha_{2}} \cdots p_{k}^{\alpha_{k}}$ be the factorization of $q$ into prime powers, then $\alpha_{i} \geq 2, i=1,2, \ldots, k$. Then for any integer $n$ with $(n, q)=1$, from Lemma 3, Lemma 4 and the properties of Dirichlet character we have

$$
\begin{aligned}
\sum_{\chi \bmod q}|G(n, \chi ; q)|^{6} & =\prod_{p^{\alpha} \| q}\left[\sum_{\chi \bmod p^{\alpha}}\left|G\left(n q / p^{\alpha}, \chi ; p^{\alpha}\right)\right|^{6}\right] \\
& =\prod_{p^{\alpha} \| q}\left[8 \phi^{2}\left(p^{\alpha}\right) p^{2 \alpha}\right] \\
& =8^{\omega(q)} \cdot q^{2} \cdot \phi^{2}(q),
\end{aligned}
$$

where $\prod_{p^{\alpha} \| q}$ denotes that $p^{\alpha} \mid q$ and $p^{\alpha+1} \nmid q$.

This completes the proof of Theorem 1 .

Acknowledgement. The author expresses her gratitude to the referee for her very helpful and detailed comments improving this paper.

\section{References}

[1] Tom M. Apostol, Introduction to Analytic Number Theory, Springer-Verlag, New York, 1976.

[2] T. Cochrane and Z. Y. Zheng, Pure and mixed exponential sums, Acta Arith. 91 (1999), no. $3,249-278$.

[3] L. K. Hua, Introduction to Number Theory, Science Press, Beijing, 1979.

[4] A. Weil, On some exponential sums, Proc. Nat. Acad. Sci. U.S.A. 34 (1948), 204-207.

[5] W. Zhang, Moments of generalized quadratic Gauss sums weighted by L-functions, J. Number Theory 92 (2002), no. 2, 304-314.

[6] W. Zhang and H. Liu, On the general Gauss sums and their fourth power mean, Osaka J. Math. 42 (2005), no. 1, 189-199.

YANFENG HE

Department of Mathematics

NORTHWEST UNIVERSITY

Xi'An, ShaAnXi 710069, P. R. China

AND

College of Mathematics and Computer Science

YAN'AN UNIVERSITY

YAN'AN, ShaAnXi, 716000, P. R. China

E-mail address: ydheyanfeng@gmail.com

WENPENG Zhang

Department of Mathematics

NORTHWEST UNIVERSITY

Xi'An, ShaAnxi 710069, P. R. China

E-mail address: wpzhang@nwu.edu.cn 